\title{
Intravenous antibiotics for the treatments of cholangitis after Kasai operation in biliary atresia patients
}

\section{Pei Wang}

Tongji Hospital of Tongji Medical College of Huazhong University of Science and Technology

Dandan Li

Tongji Hospital of Tongji Medical College of Huazhong University of Science and Technology

Chunlei Jiao

Tongji Hospital of Tongji Medical College of Huazhong University of Science and Technology

\section{Didi Zhuansun}

Tongji Hospital of Tongji Medical College of Huazhong University of Science and Technology

\section{Ying $\mathrm{He}$}

Tongji Hospital of Tongji Medical College of Huazhong University of Science and Technology

Jiexiong Feng ( $\nabla$ fengjiexiong1020@163.com )

Tongji Hospital of Tongji Medical College of Huazhong University of Science and Technology https://orcid.org/0000-0001-5008-6170

\section{Research article}

Keywords: Biliary atresia, Kasai portoenterostomy, Cholangitis, Intravenous antibiotics

Posted Date: April 7th, 2020

DOl: https://doi.org/10.21203/rs.3.rs-20701/v1

License: (9) This work is licensed under a Creative Commons Attribution 4.0 International License. Read Full License 


\section{Abstract}

Objective: This is a single-center, retrospective study, to assess the experimental treatment of intravenous antibiotics for cholangitis after Kasai portoenterostomy (PKE) in biliary atresia (BA) patients.

Methods: 153 cholangitis patients after PKE from January 2016 to December 2018 were retrospectively reviewed. Based on the different kinds of antibiotics treatment, all patients were divided into 3 groups: Intravenous Cefoperazone Sodium Tazobactam Sodium (ICSTS) group, Intravenous Meropenem (IMP) group, Meropenem combined with Intravenous immunoglobulin (IVIG) group, compared the tre atment effect among 3 groups.

Results: The recurrent cholangitis rate of ICSTS group, IMP group and IVIG group is $28.9 \%, 39.4 \%, 85.7 \%$ respectively. The IVIG group had higher temperature (T), white blood cell (WBC), C-reactive protein (CRP), alanine transaminase (ALT) and aspartate transaminase (AST) lever than ICSTS group and IMP group (P $<0.05)$ before interference. The antifebrile rate had no significant difference among 3 groups after 3 days hospitalization, as we expected, the level of WBC, CRP, DBL was significant descend in each group, the level of ALT, AST, Y-GGT was obviously decline in IVIG group. After 3 months followed-up, IVIG group showed a recurrent rate of $82 \%$, which was higher than ICSTS group and IMP group ( $P=0.013)$, the cholangitis frequency was significant higher in IVIG group than ICSTS group and IMP group $(P<0.05)$.

Conclusions: Personalized intravenous antibiotics to treat cholangitis considering recurrent episode, the severity of infection and liver function, may be a more appropriate treatment strategy.

\section{Introduction}

Biliary atresia (BA) is a serious neonatal disease characterized by intrahepatic and extrahepatic biliary system obstruction, progressive jaundice and irreversible fibrosis (1). Timely Kasai Portoenterostomy (PKE) following with reasonable perioperative managements had improved the prognosis, liver transplantation was still necessary to majority BA patients for long-term survival, which is the most common reason for pediatric liver transplantation (LT) worldwide (2).

Cholangitis is the most common complication in post-Kasai BA patients, and its incidence is about $50 \% \sim 90 \%(3-5)$. The pathogenesis is not unified, and most widely believed mechanism is an ascending infection by bacteria in the bowel, immigrated into the portal venous system. Early cholangitis and recurrent episode are important risk factors affecting the postoperative life quality of BA patients, leading to an increasing worsened prognosis and mortality (5). Therefore, timely and effective therapeutic is significant to cure cholangitis.

In recent years, third-generation of cephalosporins and carbapenem antibiotics are both the first-line drugs in the treatment of cholangitis patients after PKE (6). However, patients with grievous or intractable cholangitis does not respond to the usual treatment, Intravenous immunoglobulin (IVIG) may be considered as add-on treatment to prevent the progressive of cholangitis, minimize the damage to the 
biliary drainage (7). In this study, we particularly investigate the intravenous antibiotics treatment for cholangitis patients post-Kasai operation.

\section{Patients And Methods}

\section{Patients}

A retrospective study was carried out for all cholangitis patients after PKE hospitalized at the Department of Pediatric Surgery, Tongji Hospital during January 2016 to December 2018. All patients were unreceived any additional antibiotics treatment before enrolling. An episode of postoperative cholangitis was defined as the presence of unexplained fever $\left(\geq 38^{\circ} \mathrm{C}\right)$ combine with at least one of the following findings: $\left.\mathrm{i}\right)$ clinical symptoms such as reappearance jaundice or acholic stool; ii) increased serum direct bilirubin level ( $\mathrm{DBL} \geq 20 \mu \mathrm{mol} / \mathrm{L}$ ); iii) elevated white blood cell (WBC) or C-reactive protein (CRP).

\section{Methods}

All patients were received one kind of following intravenous antibiotics treatments: i) intravenous Cefoperazone Sodium Tazobactam Sodium (ICSTS) at $100 \mathrm{mg}^{\mathrm{kg}}{ }^{-1}$, Bid, ii) intravenous meropenem (IMP) at $20 \mathrm{mg} \cdot \mathrm{kg}^{-1}, \mathrm{Q} 8 \mathrm{~h}$, iii) IVIG at $400 \mathrm{mg} \cdot \mathrm{kg}^{-1}$. day ${ }^{-1}$ for 3 days combined with meropenem at 20 mg. $\mathrm{kg}^{-1}, \mathrm{Q} 8 \mathrm{~h}$. The therapeutic strategy of each cholangitis patient was considered the severity of cholangitis and decided by at least 3 pediatric surgeons, informed consents were obtained from all patients' parents before intervened. All patients were accepted adjuvant treatment as follows: intravenous metronidazole at $20 \mathrm{mg} \cdot \mathrm{kg}^{-1}$.day ${ }^{-1}$, oral prednisone at $5 \mathrm{mg} \cdot \mathrm{kg}^{-1}$.day ${ }^{-1}$ for 5 days, then taper down over a week, oral ursodesoxycholic acid at $10 \mathrm{mg} \cdot \mathrm{kg}^{-1}$, bid.

Based on the intravenous antibiotic treatments, all patients were divided into 3 groups: ICSTS group, IMP group and IVIG group. patients' personal information was consisted of gender, age, body weight, age at PKE, age at the first episode of cholangitis, episodes of cholangitis. Besides, we recorded patients' body temperature $(T), W B C, C R P$, alanine transaminase (ALT) and aspartate transaminase (AST), $Y^{-}$ Gltamyltranspeptidase (Y-GGT) and DBL before interfered, we would thereby compare the patients' information, liver function tests, infection marker among the 3 groups. Then collected the antifebrile rate and hospital stay lengths to compare with each other. WBC, CRP, ALT, AST, Y-GGT, DBL after 3 days' treatment were recorded and given before-and-after comparison. All patients were followed up for 3 months or until they reached an end point (liver transplantation or death), calculated the recurrent episodes of cholangitis during the 3 months and contrasted to each other.

\section{Ethics}

The Institutional Review Board of Tongji Hospital, Tongji Medical College, Huazhong University of Science and Technology approved the protocol of the study (Permit Number 2010-HP0761, Wuhan, China). All procedures performed in studies involving human participants were in accordance with the 
ethical standards of the institutional and/or national research committee and with the 1964 Helsinki declaration and its later amendments or comparable ethical standards.

\section{Statistical analysis}

All statistical analyses were performed using IBM SPSS Statistics version 26. Date were presented as mean \pm standard error of the mean (SEM) or percentage. The Kruskal-Wallis H-test was used to compare the difference among the 3 groups. The Student's $t$ test was used for the before-and-after comparison in each group. The Chi-square (Fisher's exact) test was used for comparisons of cholangitis episodes and antifebrile rate. A $P$ value of $<0.05$ was considered statistically significant.

\section{Results}

\section{Patients' information}

We enrolled 153 cholangitis patients after PKE from January 2016 to December 2018, and 45 patients belonged to ICSTS group, 94 patients belonged to IMP group, 14 patients belonged to IVIG group. Patients' personnel information was analyzed and is displayed in table 1. Age of ICSTS group, IMP group, IVIG group was $11.11 \pm 2.77$ months, $5.12 \pm 0.32$ months, $7.96 \pm 1.37$ months corresponding. Age at KPE of IVIG group was the eldest among 3 groups $(P=0.020)$, age at the first cholangitis onset was youngest in IVIG group, $(P=0.018)$, patients in IVIG group had a significant highest cholangitis frequency (3.57 \pm 0.43 times, $P=0.011)$ and recurrent rate $(85.7 \%, P=0.019)$ before interfere.

\section{Infection markers and liver function tests before interference}

Infection markers and liver function tests are displayed in table 2, the $T$ of the 3 groups was $38.18 \pm 0.09^{\circ} \mathrm{C}, 38.78 \pm 0.06^{\circ} \mathrm{C}, 39.45 \pm 0.23^{\circ} \mathrm{C}$ corresponding and has significant difference with each other $(P<0.001)$, IVIG group had the highest WBC in 3 groups $\left(21.01 \pm 1.16 \times 10^{9} / L, P=0.013\right)$, the CRP was the lowest in ICSTS group $(40.06 \pm 4.37 \mathrm{mg} / \mathrm{L})$ and the highest in IVIG group $(122.39 \pm 18.65 \mathrm{mg} / \mathrm{L}, \mathrm{P}=0.001)$. Compared with ICSTS group and IMP group, IVIG group had the highest level in ALT $(P=0.004)$ and AST $(P=0.019)$. Similarly, compared with IMP group and IVIG group, ICSTS group had the lowest level in $Y^{-}$ GGT $(P=0.041)$ and DBL $(P=0.015)$.

\section{Before-and-after comparison of each group}

Affection markers and liver function of all cholangitis patients were recorded after 3 days treatment. In ICSTS group (table 2), Inflammatory biomarkers, including WBC and CRP, showed significantly difference before-and-after treatment. WBC level was lower $\left(12.33 \pm 0.93 \times 10^{9} / \mathrm{L}\right)$ compared with un-treatment $\left(15.29 \pm 0.97 \times 10^{9} / \mathrm{L}\right)(\mathrm{P} 1=0.030)$. CRP was significantly declined before-and-after comparison $(40.06 \pm 4.37 \mathrm{mg} / \mathrm{L}$ vs $10.60 \pm 2.65 \mathrm{mg} / \mathrm{L}, \mathrm{P} 1<0.05)$. The level of $\mathrm{DBL}$ was significantly descend after treatment $(45.96 \pm 3.76 \mu \mathrm{mol} / \mathrm{L}$ vs $33.96 \pm 3.67 \mu \mathrm{mol} / \mathrm{L}, \mathrm{P} 1=0.025)$. ALT, AST and $\mathrm{Y}-\mathrm{GGT}$ had a trend of decreased, but the difference was no significance. In IMP group (table 2), it showed a similar trend with 
ICSTS group, the level of WBC, CRP and DBL was sharply declined after 3 days treatment and the difference was significant. In IVIG group (table 2), the therapeutic efficacy was especially effectively, patients showed a significant different level of WBC before-and-after comparison $\left(21.01 \pm 1.16 \times 10^{9} / \mathrm{L}\right.$ vs $\left.11.82 \pm 1.41 \times 10^{9} / \mathrm{L}, \mathrm{P} 3<0.05\right), \mathrm{CRP}$ was significantly declined after treatment $(122.39 \pm 18.65 \mathrm{mg} / \mathrm{L}$ vs $24.87 \pm 5.59 \mathrm{mg} / \mathrm{L}, \mathrm{P} 3<0.05)$, the liver function including $A L T, A S T$ and $\gamma-G G T$, all had taken a turn for the better, and the change showed significantly. The level of DBL was also significantly descend after treatment $(88.96 \pm 12.02 \mu \mathrm{mol} / \mathrm{L}$ vs $65.26 \pm 10.25 \mu \mathrm{mol} / \mathrm{L}, \mathrm{P} 3<0.05)$.

\section{Patients' outcomes}

After 3 days hospitalization, the 3 groups all had a satisfied antifebrile rate ( $82.2 \%$ vs $80 \%$ vs $92.9 \%)$, and has no significant difference with each other $(P=0.589)$. On the aspect of hospital stay lengths, there was no significant difference among the 3 groups $(P=0.559)$. During the lasting 3 months follow-up, 2 patients were given LT in ICSTS group, 3 patients were recorded adverse outcomes in IMP group, which are 1 LT and 2 death, in IVIG group, there was also $1 \mathrm{LT}$ and 2 death, and the adverse outcomes rate were $4.4 \%$, $3.6 \%, 21.4 \%$ respectively, showed a significant difference compared IVIG group with the other 2 groups $(P=0.031)$. After 3 months followed-up, IVIG group showed more likely to experience recurrent episode of cholangitis and the rate was $82 \%$, which was higher than the other 2 groups $(P=0.013)$. On the aspect of cholangitis frequency during the 3 months, patients in IVIG group had a significant higher cholangitis frequency than ICSTS group and IMP group $(P=0.004)$.

\section{Discussion}

In this retrospective study, we analyzed the therapeutic efficacy of intravenous multiple antibiotics for cholangitis, based on a before-and-after comparison of 153 cholangitis patients after PKE procedure. To investigate the severity of cholangitis and the related antibiotics treatments, we further compared the difference of infection markers and liver function among the 3 groups based on the treatment of intravenous antibiotics.

Cholangitis is the most common complication after PKE procedure. In recent years, the mechanism of cholangitis has been studied, but not unified. It is generally believed that bile duct injury, bile duct dysplasia, intestinal microbial migration and retrograde infection caused by intestinal contents reflux are the main causes of cholangitis (8). When the occurrence of biliary insufficiency obstruction, bile drainage is not sufficient, intestinal dysfunction and other reasons, can cause intestinal bacteria migration and intrahepatic reproduction, eventually lead to cholangitis. Evidence supports the hypothesis that bacterial translocation from the enteric conduit is the main pathogenic mechanism (9). Reduced lymph drainage at the porta hepatis and an insufficient volume of bile flow from a partially obstructed biliary tree were recognized as possible causative factors for the bacterial translocation. It has been reported that grampositive bacteria, especially Enterococci species, occupied a significant portion of the etiologic pathogens of cholangitis after KPE. In previous studies, the most frequently isolated bacteria were $\mathrm{E}$. coli, $\mathrm{K}$. pneumoniae, and P. aeruginosa, E. cloacae. Enterococcus faecium is a well-known intestinal bacterium 
that is known to cause acute cholangitis in adults, Baek SH et al. reported that Enterococci faecium had a high prevalence rate in culture-proven cholangitis, but easily development to antimicrobial resistance, which demonstrates that they may be considered as a pathogen of recurrent cholangitis and intractable cholangitis after KPE (8).

In present, many researches divided cholangitis into early cholangitis and late cholangitis. Early cholangitis is associated with edema, necrosis and granulation tissue proliferation at the anastomotic stoma, which will lead to the small bile duct obstruction. The late cholangitis is more concerned with the progressive damage of small bile ducts due to the abnormal development of the bile duct in the patients (9). Several previous studies have demonstrated that early cholangitis is associated with a poor prognosis $(4,5)$. In our study, the patients' age in ICSTS group is elder than IMP and IVIG groups, we further discover that age at the first episode cholangitis is eldest in ICSTS group, indicates that the elder age of the patients, the milder of cholangitis, tend to have a better outcome, though it's just a rough trend. It was common acknowledged that an elder age at KPE usually indicates a potential for more severe liver damage and poor outcomes (10), though no study has demonstrated the relation between surgical age and cholangitis, in our study, we find out that IVIG group trend to have elder surgical age than the other two groups. Recurrent episodes of cholangitis result in inflammatory reaction, bile duct injury and stenosis, lead to poor bile drainage can finally cause intrahepatic bile duct cystic dilation to form bile lake, which is also a risk factor for another episode cholangitis and a sign of poor prognosis (4). In our study, we assessed the frequency and recurrent episodes of cholangitis in 3 groups, the IVIG group shows a highest cholangitis frequency ( $3.57 \pm 0.43$ times) and the recurrent rate is $85.7 \%$, significantly higher than the other two groups, which is consistent with previous studies that recurrent cholangitis resulting in poor prognosis.

Cholangitis is the most common complication in BA patients after KPE, the incidence is about $50 \% \sim 90 \%$. It has reported that varies regimes for prevention of cholangitis in patients with BA after KPE, prophylactic antibiotics after KPE to reduce the incidence of cholangitis is universal, but few published studies provide satisfactory evidence for its benefit ${ }^{[11]}$. furthermore, the latest research, Wen Bo Pang et al. reported that prophylactic imipenem-cilastatin and human immunoglobulin is effectively on the prevention of earlyonset cholangitis (12). Although the use of steroids plays active role in bile flow after Kasai procedures, there was no evidence that it plays any role on prevention of cholangitis. There is no debate that cholangitis is the most common risk factor influence the quality of life in KPE patients. Each episode of cholangitis would damage the liver function, accelerate liver fibrosis, and lead to poor prognosis, therefore, an early diagnosis and timely antimicrobial management are important to prevent liver persistent damage caused by cholangitis $(3,4)$.

Up to now, no studies had a standard protocol for the evaluation of suspected ascending cholangitis, most reported that the diagnosis was based on clinical presentation and liver biochemistries: unexplainable fever, recurrent jaundice or acholic stools, increased bilirubin levels, elevated WBC and CRP (6). Blood culture can confirm detect the pathogenic bacteria causing cholangitis, but the positive rate is very low, ranging from $8.9-35.1 \%(8,13,14)$. It was reported that liver biopsy could be considered to obtain 
liver tissue sample for bacterial culturing, however, it is not easy to perform in children owing to its invasiveness. Some rare diagnostic methods include: bile culture is invasive, and liver puncture is not guaranteed to take bile unless there is a definite bile lake (15).

When a patient after PKE procedure is suffering from an episode of cholangitis, which antibiotics are the best choice? During the last three decades, the type of antibiotics used for cholangitis after PKE has changed over time. In the early 1980s, second-generation cephalosporins (cefuroxime and cefamandole) with or without aminoglycoside (gentamicin and amikacin) were commonly used. Since 1989, thirdgeneration cephalosporins (usually cefoperazone) were widely used. In 2004, meropenem was introduced as a suitable candidate, suggested became an effective first-line antibiotic. Unfortunately, the efficacy of antibiotics in the treatment of cholangitis PKE decreased as time goes on (3). The probably reasons are following, Unreasonably and irregularly applied antibiotics contribute to pharmaceutical resistance,the pathogenic bacteria was variable and easily development to antimicrobial resistance. Furthermore, recurrent cholangitis lead to serious hepatic hilum fibrosis and the drug concentration is not enough.

Each episode of cholangitis would damage the liver function, accelerate liver fibrosis, and lead to various complications, at the same time, in order to treatment cholangitis, a variety of drugs will increase the burden of the liver, affect the prognosis of children. Previous studies have suggested that recurrent episode and inadequately treated cholangitis could lead to progressive liver failure (16). Therefore, judicious empiric antimicrobial management are, therefore, important to prevent liver damage caused by cholangitis and the emergence of multi-drug-resistant organisms. In this study, IVIG group had a significant high level of $\mathrm{T}\left(39.45 \pm 0.23^{\circ} \mathrm{C}\right)$ and WBC $\left(21.01 \pm 1.16 \times 10^{9} / \mathrm{L}\right)$, as well as CRP $(122.39 \pm$ $18.65 \mathrm{mg} / \mathrm{L})$, the liver function including ALT, AST, $\mathrm{Y}$-GGT and DBL were significant elevated and higher than the other two groups. We indicate that intravenous antibiotics were more likely to consider the infection markers and liver function, a patient with serious infection and worse liver function was prefer given higher level intravenous, such as IMP, even more combined with IVIG.

Third-generation cephalosporins (usually cefoperazone) and meropenem are dominant in treating cholangitis, however, recent study showed that more than half of cholangitis patient treatment with meropenem are invalid and eventually required a switch of antibiotics, indicated that meropenem alone may no longer be the most appropriate antibiotics. An early switch or addition of antibiotics should be considered in intractable cholangitis to minimize the damage to the biliary drainage (3). It has been reported that infants with BA always experience immune dysregulation and deficiency in addition to inflammatory conditions. IVIG is known to reduce inflammatory cytokines and to increase antiinflammatory regulatory $T$ cells. Numerous mechanisms for the anti-inflammatory action of IVIG have been proposed, including interference with the cytokine network, neutralization of autoantibodies, modulation of effector functions of $\mathrm{T}$ and $\mathrm{B}$ cells, and enhancement of regulatory $\mathrm{T}$ cells. In addition, human immunoglobulin reduces progressive intra-hepatic bile duct injury. The previous studies have shown that supplementation of IVIG with severe infection rapidly improved the immune function and antiinfection ability of the children, and reduced the development of the systemic inflammatory response syndrome, previous study considered of IVIG use in infants with BA to diminish the progressive 
intrahepatic bile duct injury (17-19). Our previous study showed that IVIG as add-on treatment may be an effective treatment for the cholangitis acute episode (7). In this study, after three days treatment, the three groups all attained relatively high antifebrile rate of $82.2 \%, 80 \%$ and $92.9 \%$, and hospital stay length had no significant difference, indicate that the three groups patients all obtained satisfactory therapeutic efficiency, though IVIG group displayed more serious infection and worse liver function before treatment. With three months followed up, unfortunately, IVIG group had a significant higher recurrent rate of $82 \%$ compared with ICSTS group (23\%) and IMP group (37\%), the cholangitis frequency is $1.36 \pm 0.28$ times in IVIG group, which is the most frequent group. Furthermore, IVIG group also had a worse adverse outcomes rate $(21.4 \%, 2$ death and $1 \mathrm{LT})$, significantly higher than the others. The reason may be that supplementation of IVIG with severe infection rapidly improved the immune function, but the duration seems to be limited, serious and intractable cholangitis PKE tend to has a poor prognosis.

However, our study had limitations and shortages. It was a retrospective study with small sample, which may have a slight deviation, Prospective study is needed to verify it. In this study, we didn't analyze the blood culture, for not all patients received blood culture and the positive rate is low. We just analyze the outcome of the following 3months, long-term follow-up should be further recorded. IVIG is not a routine treatment for cholangitis, we would obtain parental consent before application of IVIG and all the patients had equal rights to given the best treatment.

There is no unified guideline treatment of intravenous antibiotics for cholangitis PKE, IVIG as supplementary treatment may improve short-outcome of serious and intractable cholangitis. Personalized intravenous antibiotics to treat cholangitis considering recurrent episode, the severity of infection and liver function, may be a more appropriate treatment strategy. A multi-center prospective study should be studied to provide a standard intravenous antibiotics treatment of cholangitis.

\section{Declarations}

\section{Authors' Contributions}

Jiexiong Feng and Pei Wang conceptualized and designed the study. Pei Wang drafted the initial manuscript. Pei Wang, Dandan Li and Chunlei Jiao collected patient data in a database, Didi Zhuansun and Ying He carried out statistical analyses. All authors approved the final manuscript as submitted and agree to be accountable for all aspects of the work.

\section{Funding}

This work was supported by 1. National Natural Science Foundation of China (no. 81873541);2. National Key Research and Development Program of China [grant number: 2016YFE0203900].

\section{Availability of data and materials}

The data is available from the corresponding author on reasonable request. 


\section{Conflict of interest}

The authors declare that they have no conflict of interest.

\section{References}

1. Qiao G, Li L, Cheng W, et al. Conditional probability of survival in patients with biliary atresia after Kasai portoenterostomy: a Chinese population-based study. J Pediatr Surg. 2015;50(8):1310-5. doi:10.1016/j.jpedsurg.2015.03.062.

2. Superina R, Magee JC, Brandt ML, et al. The anatomic pattern of biliary atresia identified at time of Kasai hepatoportoenterostomy and early postoperative clearance of jaundice are significant predictors of transplant-free survival. Ann Surg. 2011;254(4):577-85.

doi:10.1097/SLA.0b013e3182300950.

3. Chung PHY, Wong KKY, Tam PKH. Does the identity of the bacteria matter in post-Kasai cholangitis? A comparison between simple and intractable cholangitis. J Pediatr Surg. 2018;53(12):2409-11. doi:10.1016/j.jpedsurg.2018.08.028.

4. Chen SY, Lin CC, Tsan YT, et al. Number of cholangitis episodes as a prognostic marker to predict timing of liver transplantation in biliary atresia patients after Kasai portoenterostomy. BMC Pediatr. 2018;18(1):119. doi:10.1186/s12887-018-1074-2.

5. Liu J, Dong R, Chen G, et al. Risk Factors and Prognostic Effects of Cholangitis after Kasai Procedure in Biliary Atresia Patients: A Retrospective Clinical Study. J Pediatr Surg. 2019;54(12):2559-64. doi:10.1016/j.jpedsurg.2019.08.026.

6. Luo Y, Zheng S. Current concept about postoperative cholangitis in biliary atresia. World J Pediatr. 2008;4(1):14-9. doi:10.1007/s12519-008-0003-0.

7. Li D, Wang P, He Y, et al. Intravenous immunoglobulin for the treatment of intractable cholangitis after Kasai portoenterostomy in biliary atresia patients. Pediatr Surg Int. 2018;34(4):399-404. doi:10.1007/s00383-018-4240-6.

8. Baek SH, Kang JM, Ihn K, et al. The Epidemiology and Etiology of Cholangitis after Kasai Portoenterostomy in Patients with Biliary Atresia. J Pediatr Gastroenterol Nutr. 2019. doi:10.1097/MPG.0000000000002555. [Epub ahead of print].

9. Yang LY, Fu J, Peng XF, et al. Validation of aspartate aminotransferase to platelet ratio for diagnosis of liver fibrosis and prediction of postoperative prognosis in infants with biliary atresia. World $\mathrm{J}$ Gastroenterol. 2015;21(19):5893-900. doi:10.3748/wjg.v21.i19.5893.

10. Chardot C, Carton M, Spire-Bendelac N, et al. Is the Kasai operation still indicated in children older than 3 months diagnosed with biliary atresia? J Pediatr. 2001;138(2):224-8.

11. Decharun K, Leys CM, West KW, et al. Prophylactic Antibiotics for Prevention of Cholangitis in Patients With Biliary Atresia Status Post-Kasai Portoenterostomy: A Systematic Review. Clin Pediatr (Phila). 2016;55(1):66-72. doi:10.1177/0009922815594760. 
12. Pang WB, Zhang TC, Chen YJ, et al. Ten-Year Experience in the Prevention of Post-Kasai Cholangitis. Surg Infect (Larchmt). 2019;20(3):231-5. doi:10.1089/sur.2018.047.

13. Ernest van Heurn LW, Saing H, Tam PK. Cholangitis after hepatic portoenterostomy for biliary atresia: a multivariate analysis of risk factors. J Pediatr. 2003;142(5):566-71.

14. Wu ET, Chen HL, Ni YH, et al. Bacterial cholangitis in patients with biliary atresia: impact on shortterm outcome. Pediatr Surg Int. 2001;17(5-6):390-5.

15. Mavilia MG, Molina M, Wu GY. The Evolving Nature of Hepatic Abscess: A Review. J Clin TransI Hepatol. 2016;4(2):158-68. doi:10.14218/JCTH.2016.00004.

16. Chung PH, Wong KK, Tam PK. Predictors for failure after Kasai operation. J Pediatr Surg. 2015;50(2):293-6. doi:10.1016/j.jpedsurg.2014.11.015.

17. Roopenian DC, Akilesh S. FcRn: the neonatal Fc receptor comes of age. Nat Rev Immunol. 2007;7(9):715-25.

18. Tha-In T, Bayry J, Metselaar HJ, et al. Modulation of the cellular immune system by intravenous immunoglobulin. Trends Immunol. 2008;29(12):608-15. doi:10.1016/j.it.2008.08.004.

19. Durandy A, Kaveri SV, Kuijpers TW, et al. Intravenous immunoglobulins-understanding properties and mechanisms. Clin Exp Immunol. 2009;158(Suppl 1):2-13. doi:10.1111/j.13652249.2009.04022.x.

\section{Tables}

\begin{tabular}{lllll}
\hline Table 1 Patients information & \multicolumn{3}{c}{ IVIG } & \multicolumn{2}{c}{ P value } \\
\hline Age(months) & ICSTS & \multicolumn{1}{c}{ IMP } & \multicolumn{2}{c}{$0.08^{*}$} \\
Kasai age(days) & $11.11 \pm 2.77$ & $5.12 \pm 0.32$ & $7.96 \pm 1.37$ & $0.020^{*}$ \\
AFC (months) & $58.80 \pm 1.65$ & $62.01 \pm 1.82$ & $74.71 \pm 4.13$ & 0.02 \\
Cholangitis frequency (times) & $1.33 \pm 0.08$ & $1.54 \pm 0.08$ & $3.57 \pm 0.43$ & $0.011^{*}$ \\
Recurrent rate (\%) & 28.9 & 39.4 & 85.7 & $0.019^{*}$ \\
\hline
\end{tabular}

ICSTS: Intravenous Cefoperazone Sodium Tazobactam Sodium, IMP: Intravenous Meropenem, IVIG: Intravenous immunoglobulin. AFC: age at the first cholangitis onset. Cholangitis frequency: including this episode of cholangitis. Recurrent rate: This episode of cholangitis is not the first one. $\mathrm{P}^{*}<0.05$. 


\begin{tabular}{|c|c|c|c|c|c|c|c|c|c|c|}
\hline & \multicolumn{3}{|c|}{ ICSTS } & \multicolumn{3}{|c|}{ IMP } & \multicolumn{3}{|c|}{ IVIG } & \multirow[b]{2}{*}{$\begin{array}{c}P \\
\text { value }\end{array}$} \\
\hline & before & after & $\begin{array}{l}\text { P1 } \\
\text { value }\end{array}$ & before & after & $\begin{array}{l}\mathrm{P} 2 \\
\text { value }\end{array}$ & before & after & $\begin{array}{l}\text { P3 } \\
\text { value }\end{array}$ & \\
\hline $\mathrm{T}\left({ }^{\circ} \mathrm{C}\right)$ & $38.18 \pm 0.09$ & 1 & 1 & $38.78 \pm 0.06$ & 1 & 1 & $39.45 \pm 0.23$ & 1 & 1 & $0.000^{*}$ \\
\hline $\begin{array}{l}\text { WBC } \\
\left(\times 10^{9} / \mathrm{L}\right)\end{array}$ & $15.29 \pm 0.97$ & $12.33 \pm 0.93$ & $0.030^{*}$ & $15.80 \pm 0.75$ & $13.31 \pm 0.56$ & $0.008^{*}$ & $21.01 \pm 1.16$ & $11.82 \pm 1.41$ & $0.000^{*}$ & $0.013^{*}$ \\
\hline $\begin{array}{l}\text { CRP } \\
(\mathrm{mg} / \mathrm{L})\end{array}$ & $40.06 \pm 4.37$ & $10.60 \pm 2.65$ & $0.000^{*}$ & $64.59 \pm 4.74$ & $23.53 \pm 3.11$ & $0.000^{*}$ & $122.39 \pm 18.65$ & $24.87 \pm 5.59$ & $0.000^{*}$ & $0.001^{*}$ \\
\hline $\begin{array}{l}\text { ALT } \\
(\mathrm{U} / \mathrm{L})\end{array}$ & $193.58 \pm 14.67$ & $\begin{array}{l}154.31 \pm 12.6 \\
2\end{array}$ & 0.054 & $180.58 \pm 13.45$ & $160.95 \pm 12.74$ & 0.293 & $298.51 \pm 34.61$ & $222.29 . \pm 30.18$ & $0.000^{*}$ & $0.004^{*}$ \\
\hline $\begin{array}{l}\text { AST } \\
(\mathrm{U} / \mathrm{L})\end{array}$ & $166.80 \pm 77.10$ & $\begin{array}{l}147.40 \pm 13.1 \\
1\end{array}$ & 0.269 & $147.12 \pm 9.83$ & $142.20 \pm 9.26$ & 0.514 & $286.19 \pm 30.87$ & $215.36 \pm 31.55$ & $0.000^{*}$ & $0.019^{*}$ \\
\hline $\begin{array}{l}\gamma \text {-GGT } \\
(\mathrm{U} / \mathrm{L})\end{array}$ & $787.64 \pm 89.95$ & $\begin{array}{l}757.55 \pm 87.5 \\
7\end{array}$ & 0.811 & $\begin{array}{l}1019.97 \\
\pm 60.98\end{array}$ & $\begin{array}{l}1035.59 \\
\pm 63.95\end{array}$ & 0.782 & $\begin{array}{l}1275.43 \\
\pm 125.74\end{array}$ & $\begin{array}{l}1146.43 \\
\pm 122.75\end{array}$ & $0.033^{*}$ & $0.041^{*}$ \\
\hline $\begin{array}{l}\mathrm{DBL} \\
(\mu \mathrm{mol} / \mathrm{L})\end{array}$ & $45.96 \pm 3.76$ & $33.96 \pm 3.67$ & $0.025^{*}$ & $70.15 \pm 4.69$ & $58.42 \pm 4.59$ & $0.005^{*}$ & $88.96 \pm 12.02$ & $65.26 \pm 10.25$ & $0.000^{*}$ & $0.015^{*}$ \\
\hline
\end{tabular}

T: temperature, WBC: white blood cell count, CRP: C reactive protein, ALT: alanine transaminase, AST: aspartate transaminase, $\gamma$-GGT: $\gamma$ Gltamyltranspeptidase, DBL: direct bilirubin.

Before: cholangitis patients before interference. after: cholangitis patients after 3 days treatment.

P1, P2, P3: each group was given before-and-after comparison. p: comparison of the clinical and laboratory findings among 3 groups before interference.

$\mathrm{P}^{*}<0.05$.

\begin{tabular}{|c|c|c|c|c|}
\hline & ICSTS & IVIG & $\mathrm{P}$ value & \\
\hline antifebrile rate (\%) & 82.2 & 80 & 92.9 & 0.589 \\
\hline Hospital stay (days) & $11.20 \pm 0.62$ & $10.68 \pm 0.54$ & $9.93 \pm 0.90$ & 0.559 \\
\hline Recurrent rate (\%) & 23 & 37 & 82 & $0.013^{*}$ \\
\hline Recurrent frequency (times) & $0.26 \pm 0.08$ & $0.47 \pm 0.07$ & $1.36 \pm 0.28$ & $0.004^{*}$ \\
\hline adverse outcomes & LT: 2 cases & $\begin{array}{l}\text { Death: } 2 \text { cases } \\
\text { LT: } 1 \text { case }\end{array}$ & $\begin{array}{l}\text { Death: } 2 \text { cases } \\
\text { LT: } 1 \text { case }\end{array}$ & - \\
\hline adverse outcomes rate (\%) & 4.4 & 3.6 & 21.4 & $0.031^{*}$ \\
\hline
\end{tabular}

antifebrile rate: was recorded after 3 days' treatment. Recurrent rate: occurred the other episode of cholangitis during the following 3 months. Recurrent frequency: frequency of cholangitis during the following 3 months.

$\mathrm{P}^{*}<0.05$.

\section{Figures}


153 cholangitis patients after PKE

from January 2016 to December 2018

Based on the intravenous antibiotic treatments

ICSTS group:
45patients

IMP group:
94patients

IVIG group:

14patients
Recorded patients' gender, age, body weight, age at PKE, age at the first episode of cholangitis, episodes of cholangitis.

Recorded T, WBC, CRP, ALT AST, $\gamma$-GGT and DBL before interfered.

Compare above information among the 3 groups.

Re-examineliver T, WBC, CRP, ALT AST, $\gamma$ -

GGT and DBL after 3 days' treatment, and was given before-and-after comparison each group.

Comparison of the antifebrile rate, hospital stay lengths among 3 groups after treatments.

Comparison of the outcomes, recurrent rate and frequency among 3 groups after 3 months follow-up.

\section{Figure 1}

A caption was not provided in this version of the paper. 